Microscopic approach to entropy production

This article has been downloaded from IOPscience. Please scroll down to see the full text article.

2013 J. Phys. A: Math. Theor. 46355003

(http://iopscience.iop.org/1751-8121/46/35/355003)

View the table of contents for this issue, or go to the journal homepage for more

Download details:

IP Address: 134.99.64.185

The article was downloaded on 15/08/2013 at 09:26

Please note that terms and conditions apply. 


\title{
Microscopic approach to entropy production
}

\author{
Raphael Wittkowski $^{1,3}$, Hartmut Löwen ${ }^{1}$ and Helmut R Brand ${ }^{2}$ \\ ${ }^{1}$ Institut für Theoretische Physik II, Weiche Materie, Heinrich-Heine-Universität Düsseldorf, \\ D-40225 Düsseldorf, Germany \\ 2 Theoretische Physik III, Universität Bayreuth, D-95540 Bayreuth, Germany \\ E-mail: raphael.wittkowski@ed.ac.uk
}

Received 20 May 2013, in final form 24 July 2013

Published 12 August 2013

Online at stacks.iop.org/JPhysA/46/355003

\begin{abstract}
It is a great challenge of nonequilibrium statistical mechanics to calculate entropy production within a microscopic theory. In the framework of linear irreversible thermodynamics, we combine the Mori-Zwanzig-Forster projection operator technique with the first and second law of thermodynamics to obtain microscopic expressions for the entropy production as well as for the transport equations of the entropy density and its time correlation function. We further present a microscopic derivation of a dissipation functional from which the dissipative dynamics of an extended dynamical density functional theory can be obtained in a formally elegant way.
\end{abstract}

PACS numbers: 82.70.Dd, 47.57.J-, 05.70.Ln

\section{Introduction}

One of the central challenges of statistical mechanics is to calculate and predict the entropy of a given system under well-specified conditions from a microscopic point of view. In equilibrium, this problem dates back to Boltzmann and it is by now standard textbook knowledge that the entropy can be obtained by a suitable phase-space averaging in different ensembles [1]. The same problem, however, is much more complicated and in general unsolved in a nonequilibrium situation: while the second law of thermodynamics predicts a global entropy production in a closed system, it is very difficult-even in principle- to calculate the entropy production within a microscopic approach that starts from the individual interactions of the particles. Linear irreversible thermodynamics [1-3] provides a framework in which this problem can be addressed systematically in a simpler way. Moreover, the Mori-Zwanzig-Forster projection operator technique (MZFT) [4-10], when supplemented with a selection of relevant slow variables, can be used to derive microscopic expressions for the dynamical evolution of these slow variables and their time correlation functions. When the MZFT is applied to the entropy density, there is, however, a principle obstacle since the entropy density does not possess any

3 Present address: SUPA, School of Physics and Astronomy, University of Edinburgh, Edinburgh, EH9 3JZ, UK. 
known microscopic operator. This fact is opposed to, for example, the particle density and the energy density, which both possess corresponding microscopic operators.

In fact, we have recently applied the MZFT in the framework of linear irreversible thermodynamics to derive a generalized (extended) dynamical density functional theory (EDDFT), which provides a microscopic basis for the dynamical evolution equations of the relevant variables [11]. The approach was only formulated for slow conserved variables such as particle and energy density. In this paper, we generalize the EDDFT approach of [11] to include the entropy density $\sigma(\vec{r}, t)$ as an additional (non-conserved) variable. Under the assumption of the local formulation of the first law of thermodynamics, one has a local relation between all relevant variables and the entropy density $[6,8,12]$ which enables one to consider the entropy density as a relevant variable. Assuming the applicability of linear irreversible thermodynamics [1-3], we derive transport equations for the entropy density as well as for its time correlation function $C_{\sigma \sigma}\left(\vec{r}, \vec{r}^{\prime}, t, t^{\prime}\right)$. The key idea here is to combine the first and second law of thermodynamics with the microscopic expressions obtained within the MZFT for the remaining slow variables in order to circumvent the obstacle that the entropy density does not possess a known microscopic operator. Thereby, we provide_-at least in principlea link of entropy production to microscopic expressions. In doing so, we also present a microscopic expression for a dissipation functional from which EDDFT [11] and in particular the traditional DDFT [13-17] can be derived in an elegant way. An important application of this reformulation of EDDFT in terms of a dissipation functional is the derivation of phase field crystal (PFC) models from EDDFT, in particular those which involve orientational degrees of freedom [18-21].

Our derivation of the transport equations for the entropy density and its time correlation function is valid for a general set of relevant variables, including variables that are not considered in the context of current EDDFT. Furthermore, the derived expressions are not restricted to the hydrodynamic limit (vanishing wave vector $\vec{k} \rightarrow \overrightarrow{0}$ and frequency $\omega \rightarrow 0$ ) and thus generalize the corresponding hydrodynamic equations to larger wave vectors and frequencies. Our approach is compatible with the so-called GENERIC formalism developed by Grmela and Öttinger [22, 23], which sets a general phenomenological framework for nonequilibrium thermodynamics. Finally, we remark that the fluctuation theorem derived by Jarzynski [24] provides another route to express entropy production in terms of microscopic quantities. The Jarzynski theorem considers the average over the work performed by the system when it goes from an initial to a final state. This is a different viewpoint and setup of entropy production as compared to our approach. In contrast to our approach, which relies on linear irreversible thermodynamics, the Jarzynski theorem is exact and therefore also applicable to situations far from thermodynamic equilibrium.

The formalism derived and presented in this paper can be applied to various problems. One important example is nonequilibrium relaxation from an initial state towards a final equilibrium state [25]. In particular, static classical DFT is capable of describing equilibrium crystals [26] and the traditional DDFT can be used to predict the dynamics of crystallization processes from an undercooled melt [27] for colloidal suspensions. For colloidal crystallization there is no latent heat since the solvent thermalizes the system instantaneously. For crystallization in molecular systems [28] or complex plasmas [29], on the other hand, latent heat is produced which slows down further crystal growth [30]. Our approach includes the corresponding entropy production upon crystallization and therefore provides an ideal microscopic framework to describe crystallization processes even in dusty plasmas [29].

This paper is organized as follows: after preliminary remarks about the considered systems and notation in section 2, we present a derivation of transport equations for the entropy density and its time correlation function as well as a microscopic expression for 
the corresponding dissipation functional in section 3. These expressions for general relevant variables are afterwards specialized to the variables of EDDFT in section 4. Finally, we conclude the paper in section 5 .

\section{Relevant variables and their transport equations}

As usual in the context of EDDFT [11], we consider a grand-canonical (total) ensemble of systems of $N$ particles with the not explicitly time-dependent Hamiltonian $\hat{H}\left(\hat{\Gamma}_{t}\right)^{4}$ and the set $\hat{\Gamma}_{t}$ of phase-space coordinates. These systems can be described by the Liouville probability density $\hat{\rho}(t)$, which is given by the solution of the Liouville-von Neumann equation

$$
\dot{\hat{\rho}}=-\hat{\mathcal{L}} \hat{\rho}=-\frac{\mathrm{i}}{\hbar}[\hat{H}, \hat{\rho}], \quad \hat{\rho}(t)=\mathrm{e}^{-\hat{\mathcal{L}} t} \hat{\rho}(0)
$$

with the Liouvillian $\hat{\mathcal{L}}$, the imaginary unit i, the reduced Planck constant $\hbar=h /(2 \pi)$, and the commutator $[X, Y]=X Y-Y X$ of $X$ and $Y$.

\subsection{Relevant variables}

Following the idea of the MZFT, we select a set of $n \ll N$ independent relevant variables (operators) $\hat{a}_{i}(\vec{r}, t)$ with $i \in\{1, \ldots, n\}$, which is sufficient to describe the considered system on a much simpler basis, where the huge number of irrelevant microscopic variables is projected out. Transport equations for the relevant variables are given by the Liouville-von Neumann equations

$$
\dot{\hat{a}}_{i}=\hat{\mathcal{L}} \hat{a}_{i}=\frac{\mathrm{i}}{\hbar}\left[\hat{H}, \hat{a}_{i}\right], \quad \hat{a}_{i}(\vec{r}, t)=\mathrm{e}^{\hat{\mathcal{L}} t} \hat{a}_{i}(\vec{r}, 0) .
$$

The set of relevant variables is associated with a relevant probability density $\rho(t)$. Together with the grand-canonical trace Tr, the relevant probability density can be used to define the averaged relevant variables

$$
a_{i}(\vec{r}, t)=\operatorname{Tr}\left(\rho(t) \hat{a}_{i}(\vec{r})\right) .
$$

We assume a given generalized Helmholtz free-energy functional $\mathcal{F}\left[a_{1}, \ldots, a_{n}\right]{ }^{5}$ that describes the state of the considered system in terms of the averaged relevant variables $a_{i}(\vec{r}, t)$. As usual in DFT, the existence of this functional is known, but its concrete form is unknown in general. The generalized Helmholtz free-energy functional $\mathcal{F}$ can be used to define the thermodynamic conjugates $^{6}$

$$
a_{i}^{\natural}(\vec{r}, t)=\frac{\delta \mathcal{F}\left[a_{1}, \ldots, a_{n}\right]}{\delta a_{i}(\vec{r}, t)}
$$

of the averaged relevant variables $a_{i}(\vec{r}, t)$. In terms of the relevant variables and their thermodynamic conjugates, the relevant probability density $\rho(t)$ is here specified as the generalized grand-canonical probability density ${ }^{7}$

$$
\rho(t)=\frac{1}{\Xi(t)} \mathrm{e}^{-\beta \hat{H}_{\text {eff }}(t)}
$$

\footnotetext{
4 For consistency, we denote all quantities that are directly associated with the set of phase-space variables $\hat{\Gamma}_{t}$ by a hat ${ }^{\wedge}$, while the hat is omitted for all other quantities.

5 The Helmholtz free-energy functional $\mathcal{F}$ is a generalization of the equilibrium Helmholtz free energy to nonequilibrium situations. See equation (6) in [11] for a microscopic definition. Static stability of the considered system requires that $\mathcal{F}$ is bounded from below.

6 The internal energy density is an exception. Its thermodynamic conjugate has to be considered differently (see sections 2.2 and 3.1).

7 See [7, 11] for further details regarding the relevant probability density $\rho(t)$. Notice that $\rho(t)$ depends on time $t$ only through the averaged relevant variables $a_{i}(\vec{r}, t)$ (see equations (4) and (6)).
} 
with the grand-canonical partition sum $\Xi(t)$, the (constant) inverse reference thermal energy $\beta=1 /\left(k_{\mathrm{B}} T_{0}\right)$, where $k_{\mathrm{B}}$ denotes the Boltzmann constant and $T_{0}$ is an absolute reference temperature $^{8}$, and the explicitly time-dependent effective Hamiltonian

$$
\hat{H}_{\text {eff }}(t)=\hat{H}-\sum_{i=1}^{n} \int_{\mathbb{R}^{3}} \mathrm{~d}^{3} r a_{i}^{\natural}(\vec{r}, t) \hat{a}_{i}(\vec{r}) .
$$

Notice that the considered system is displaced from thermodynamic equilibrium at $t=0$ so that $\rho(t)$ and $\Xi(t)$ are explicitly time-dependent.

The $n=n_{\mathrm{c}}+n_{\mathrm{n}}$ relevant variables $\hat{a}_{i}(\vec{r}, t)$ have to be distinguished into $n_{\mathrm{c}}$ conserved variables $\hat{a}_{i}^{\mathrm{c}}(\vec{r}, t)$ and $n_{\mathrm{n}}$ non-conserved variables $\hat{a}_{i}^{\mathrm{n}}(\vec{r}, t)$. The same holds for their averages $a_{i}(\vec{r}, t)$, which are distinguished into conserved averaged variables $a_{i}^{\mathrm{c}}(\vec{r}, t)$ and non-conserved averaged variables $a_{i}^{\mathrm{n}}(\vec{r}, t)$.

\subsection{Transport equations}

The dynamics of the $n_{\mathrm{c}}$ conserved relevant variables can be described by the conservation equations

$$
\dot{\hat{a}}_{i}^{\mathrm{c}}(\vec{r}, t)+\vec{\nabla}_{\vec{r}} \cdot \hat{\vec{J}}^{(i)}(\vec{r}, t)=0
$$

with the local currents $\hat{\vec{J}}^{(i)}(\vec{r}, t)$ and $i \in\left\{1, \ldots, n_{\mathrm{c}}\right\}$. Correspondingly, the dynamics of the averaged conserved relevant variables is given by

$$
\dot{a}_{i}^{\mathrm{c}}(\vec{r}, t)+\vec{\nabla}_{\vec{r}} \cdot \vec{J}^{(i)}(\vec{r}, t)=0 .
$$

On the other hand, it is assumed that the time-evolution of the $n_{\mathrm{n}}$ non-conserved relevant variables can be described by the balance equations

$$
\dot{\hat{a}}_{i}^{\mathrm{n}}(\vec{r}, t)+\hat{\Phi}^{(i)}(\vec{r}, t)=0
$$

with the local quasicurrents $\hat{\Phi}^{(i)}(\vec{r}, t)$ and $i \in\left\{1, \ldots, n_{\mathrm{n}}\right\}$, while the averaged non-conserved relevant variables are described by

$$
\dot{a}_{i}^{\mathrm{n}}(\vec{r}, t)+\Phi^{(i)}(\vec{r}, t)=0 .
$$

Both the currents $\vec{J}^{(i)}(\vec{r}, t)$ and the quasicurrents $\Phi^{(i)}(\vec{r}, t)$ can be decomposed into two different contributions [31]:

$$
\begin{aligned}
& \vec{J}^{(i)}(\vec{r}, t)=\vec{J}_{\mathrm{R}}^{(i)}(\vec{r}, t)+\vec{J}_{\mathrm{D}}^{(i)}(\vec{r}, t), \\
& \Phi^{(i)}(\vec{r}, t)=\Phi_{\mathrm{R}}^{(i)}(\vec{r}, t)+\Phi_{\mathrm{D}}^{(i)}(\vec{r}, t) .
\end{aligned}
$$

These are the reversible contributions denoted by the subscript ' $R$ ' and the dissipative contributions denoted by the subscript ' $D$ '. While the reversible contributions are isentropic, i.e., they are not associated with entropy production, the dissipative (reversible) contributions increase the total entropy of the system.

Examples for the general relevant variables $\hat{a}_{i}^{\mathrm{c}}(\vec{r}, t)$ and $\hat{a}_{i}^{\mathrm{n}}(\vec{r}, t)$ are a concentration field and a local polarization, respectively. There are only two possible relevant variables that have to be treated explicitly and cannot be taken into account by the general set $\hat{a}_{i}(\vec{r}, t)$ of relevant variables. These special variables are the conserved generalized internal energy density $\hat{\varepsilon}(\vec{r}, t)$

8 Since we are dealing with the linear response regime, a general inhomogeneous temperature field $T(\vec{r}, t)$ describes linear deviations from thermodynamic equilibrium dictated by the constant reference temperature $T_{0}$. Therefore, $\beta$ is defined as $\beta=1 /\left(k_{\mathrm{B}} T_{0}\right)$. 
or its Legendre transforms and the non-conserved entropy density $\hat{\sigma}(\vec{r}, t)$ (see section 3.1). ${ }^{9}$ Since the internal energy density is conserved, the transport equations for $\hat{\varepsilon}(\vec{r}, t)$ and $\varepsilon(\vec{r}, t)$ can be written as

$$
\begin{aligned}
& \dot{\hat{\varepsilon}}(\vec{r}, t)+\vec{\nabla}_{\vec{r}} \cdot \hat{\vec{J} \varepsilon}(\vec{r}, t)=0, \\
& \dot{\varepsilon}(\vec{r}, t)+\vec{\nabla}_{\vec{r}} \cdot \vec{J}^{\varepsilon}(\vec{r}, t)=0
\end{aligned}
$$

with the internal energy currents $\hat{\overrightarrow{J^{\varepsilon}}}(\vec{r}, t)$ and $\overrightarrow{J^{\varepsilon}}(\vec{r}, t)=\overrightarrow{J_{\mathrm{R}}^{\varepsilon}}(\vec{r}, t)+\overrightarrow{J_{\mathrm{D}}^{\varepsilon}}(\vec{r}, t)$. The entropy density, in contrast, is non-conserved and the transport equations for $\hat{\sigma}(\vec{r}, t)$ and $\sigma(\vec{r}, t)$ are

$$
\begin{aligned}
& \dot{\hat{\sigma}}(\vec{r}, t)+\hat{\Phi}^{\sigma}(\vec{r}, t)=0, \\
& \dot{\sigma}(\vec{r}, t)+\Phi^{\sigma}(\vec{r}, t)=0
\end{aligned}
$$

with the entropy quasicurrents $\hat{\Phi}^{\sigma}(\vec{r}, t)$ and $\Phi^{\sigma}(\vec{r}, t)=\Phi_{\mathrm{R}}^{\sigma}(\vec{r}, t)+\Phi_{\mathrm{D}}^{\sigma}(\vec{r}, t)$.

\subsection{Dissipation functionals}

If the considered system is in local thermodynamic equilibrium, so that the local formulation of the first and second law of thermodynamics hold, linear irreversible thermodynamics [1-3] can be applied [31]. This useful framework is especially applicable for all passive systems and states the existence of a dissipation functional $\mathfrak{R}$ from which the dissipative currents $\vec{J}_{\mathrm{D}}^{(i)}(\vec{r}, t)$ and quasicurrents $\Phi_{\mathrm{D}}^{(i)}(\vec{r}, t)$ of the averaged relevant variables $a_{i}(\vec{r}, t)$ and the entropy density $\sigma(\vec{r}, t)$ can be derived. The dissipation functional

$$
\Re=\int_{\mathbb{R}^{3}} \mathrm{~d}^{3} r \mathfrak{r}(\vec{r}, t)
$$

describes the total amount of energy that is dissipated per time unit in the considered system. Its integrand, the dissipation function $\mathfrak{r}(\vec{r}, t)$, is therefore positive semi-definite as a consequence of the second law of thermodynamics $[8,31]: \mathfrak{r}(\vec{r}, t) \geqslant 0 .{ }^{10}$ It is nonlinear in the thermodynamic variables and quadratic in the thermodynamic forces that are defined as

$$
\vec{a}_{i}^{\mathrm{c} \sharp}=-\vec{\nabla}_{\vec{r}} a_{i}^{\mathrm{c} \sharp}, \quad a_{i}^{\mathrm{n} \sharp}=a_{i}^{\mathrm{n} \downarrow}
$$

and correspond to the averaged relevant variables $a_{i}(\vec{r}, t)$. Again, the internal energy density $\varepsilon(\vec{r}, t)$ and the entropy density $\sigma(\vec{r}, t)$ have to be treated separately. While a thermodynamic force corresponding to the internal energy density does not exist, since we formulate the first law of thermodynamics in terms of the generalized internal energy as the thermodynamic potential (see equation (22) further below), the thermodynamic force $\sigma^{\sharp}(\vec{r}, t)$ corresponding to the entropy density is defined further below by equation (29). For a given dissipation functional, the dissipative currents and quasicurrents of the averaged relevant variables follow directly by functional differentiation with respect to the thermodynamic forces [31]: ${ }^{11}$

$$
\vec{J}_{\mathrm{D}}^{(i)}=\frac{\delta \Re}{\delta \vec{a}_{i}^{\mathrm{C} \sharp}}, \quad \Phi_{\mathrm{D}}^{(i)}=\frac{\delta \Re}{\delta a_{i}^{\mathrm{n} \sharp}} .
$$

9 In fact, the internal energy density $\hat{\varepsilon}(\vec{r}, t)$ has to be treated separately, since $\varepsilon(\vec{r}, t)$ is a Legendre transform of the generalized Helmholtz free-energy density that can be gradient expanded in terms of the other relevant variables. The entropy density $\hat{\sigma}(\vec{r}, t)$, on the other hand, has to be treated separately, since it is not independent of the internal energy density $\hat{\varepsilon}(\vec{r}, t)$. Both variables are related by the local formulation of the first law of thermodynamics.

${ }^{10}$ The condition $\mathfrak{r}(\vec{r}, t) \geqslant 0$ is a necessary prerequisite for dynamical stability of the considered system [31]. For entirely reversible processes, $\mathfrak{r}(\vec{r}, t)$ is zero, while it is positive for all other (dissipative) processes.

${ }^{11}$ Since the dissipation functional $\mathfrak{R}$ is quadratic in the thermodynamic forces, equation (19) implies a linear relation between the (quasi)currents and the thermodynamic forces. Notice that, in contrast, the relation between the thermodynamic forces and the relevant variables is in general strongly nonlinear. 
The dissipative energy current $\vec{J}_{\mathrm{D}}^{\vec{\varepsilon}}(\vec{r}, t)$ and the dissipative entropy quasicurrent $\Phi_{\mathrm{D}}^{\sigma}(\vec{r}, t)$ have to be derived differently (see section 3.1 further below).

For systems far from thermodynamic equilibrium, linear irreversible thermodynamics cannot be applied and a dissipation functional is in general not known [1]. This is the case for active systems like, for example, lasers, amplifiers, and biological systems. Indeed there is a generalization of the dissipation functional-the generalized Lyapunov functional-to systems that can also be far from thermodynamic equilibrium, but a Lyapunov functional does not exist in general. The criteria for the existence of a Lyapunov functional for a particular system are characterized by potential conditions [32-35].

\section{The entropy density as a relevant variable}

It is well-established in the framework of the MZFT that the entropy density is a slow variable, although it is not strictly hydrodynamic $[6,8,12]$. However, the entropy density $\sigma(\vec{r}, t)$ is a special (non-conserved) relevant variable that has not yet been considered in the context of EDDFT [11]. Although EDDFT could in principle be generalized to include non-conserved variables, the entropy density cannot be incorporated into EDDFT directly. It should instead be considered separately, since there is no corresponding operator $\hat{\sigma}(\vec{r}, t)$ known for the entropy density that would be required to incorporate the entropy density into EDDFT using the MZFT [11]. To avoid this problem, we assume the applicability of the local formulation of the first law of thermodynamics for operators and express the unknown operator for the entropy density in terms of the known operators of the other relevant variables. We thus construct an expression for the entropy density operator that is applicable in the framework of linear irreversible thermodynamics. On this basis, we present the derivation of dynamical equations for the entropy density $\sigma(\vec{r}, t)$ as well as for its time correlation function $C_{\sigma \sigma}\left(\vec{r}, \vec{r}^{\prime}, t, t^{\prime}\right)$.

\subsection{Nonequilibrium dynamics}

Since the entropy density $\sigma(\vec{r}, t)$ is not conserved, its dynamics has to be described by the balance equation (16), where the entropy quasicurrent

$$
\Phi^{\sigma}(\vec{r}, t)=\vec{\nabla}_{\vec{r}} \cdot \vec{J}^{\sigma}(\vec{r}, t)-Q^{\sigma}(\vec{r}, t)
$$

can be decomposed into a divergence term with the entropy current $\vec{J}^{\sigma}(\vec{r}, t)=\vec{J}_{\mathrm{R}}^{\sigma}(\vec{r}, t)+$ $\overrightarrow{J_{\mathrm{D}}^{\sigma}}(\vec{r}, t)$ describing the transport of entropy and a source term $Q^{\sigma}(\vec{r}, t)$ denoting the production of entropy ${ }^{12}$. The entropy production [31]

$$
Q^{\sigma}(\vec{r}, t)=\frac{2 \mathfrak{r}(\vec{r}, t)}{T(\vec{r}, t)}
$$

in turn can be expressed in terms of the dissipation function $\mathfrak{r}(\vec{r}, t)$ and the absolute local temperature $T(\vec{r}, t)$. In order to derive explicit microscopic expressions for $\vec{J}(\vec{r}, t)$ and $Q^{\sigma}(\vec{r}, t)$, we make use of the local formulation of the first law of thermodynamics ${ }^{13}[31]$

$$
\mathrm{d} \varepsilon=T \mathrm{~d} \sigma+a_{i}^{\mathrm{c} \natural} \mathrm{d} a_{i}^{\mathrm{c}}+a_{i}^{\mathrm{n} \downarrow} \mathrm{d} a_{i}^{\mathrm{n}}
$$

\footnotetext{
12 The dissipative entropy quasicurrent is therefore $\Phi_{\mathrm{D}}^{\sigma}(\vec{r}, t)=\vec{\nabla}_{\vec{r}} \cdot \vec{J}_{\mathrm{D}}^{\sigma}(\vec{r}, t)-Q^{\sigma}(\vec{r}, t)$.

${ }^{13}$ Einstein's sum convention is assumed in the following. Equation (22) makes the internal energy density $\varepsilon(\vec{r}, t)$ and the entropy density $\sigma(\vec{r}, t)$ dependent on each other and is the reason why $\varepsilon(\vec{r}, t)$ and $\sigma(\vec{r}, t)$ have to be treated separately from the other relevant variables $a_{i}(\vec{r}, t)$ (see section 2.2).
} 
with the increment of the generalized internal energy density $\mathrm{d} \varepsilon(\vec{r}, t)$, which is related to the increment of the generalized Helmholtz free-energy density $\mathrm{d} f(\vec{r}, t)$ by

$$
\mathrm{d} f=\mathrm{d} \varepsilon-T \mathrm{~d} \sigma-\sigma \mathrm{d} T,
$$

and additional conserved and non-conserved classical thermodynamic variables $a_{i}^{\mathrm{c}}(\vec{r}, t)$ and $a_{i}^{\mathrm{n}}(\vec{r}, t)$, respectively ${ }^{14}$. Equation (22) can be rearranged into

$$
\dot{\sigma}=\frac{1}{T} \dot{\varepsilon}-\frac{a_{i}^{\mathrm{c}}}{T} \dot{a}_{i}^{\mathrm{c}}-\frac{a_{i}^{\mathrm{n} \sharp}}{T} \dot{a}_{i}^{\mathrm{n}}
$$

providing a dynamical equation for the entropy density in terms of the time derivatives ${ }^{15}$ of the internal energy density and the other relevant variables. Using the transport equations (8) and (10) for the general relevant variables $a_{i}^{\mathrm{c}}(\vec{r}, t)$ and $a_{i}^{\mathrm{n}}(\vec{r}, t)$, respectively, (14) for the internal energy density $\varepsilon(\vec{r}, t)$, and (16) for the entropy density $\sigma(\vec{r}, t)$, equation (24) can be transformed into the balance equation for the entropy density (16) with the decomposition (20), the dissipative entropy current

$$
\overrightarrow{J_{\mathrm{D}}^{\sigma}}(\vec{r}, t)=\frac{1}{T} \vec{J}_{\mathrm{D}}^{\varepsilon}-\frac{a_{i}^{\mathrm{c} \natural}}{T} \vec{J}_{\mathrm{D}}^{(i)},
$$

and the entropy production

$$
Q^{\sigma}(\vec{r}, t)=-\frac{1}{T} \vec{J}_{\mathrm{D}}^{\sigma} \cdot \vec{\nabla}_{\vec{r}} T-\frac{1}{T} \vec{J}_{\mathrm{D}}^{(i)} \cdot \vec{\nabla}_{\vec{r}} a_{i}^{\mathrm{c} \sharp}+\frac{1}{T} \Phi_{\mathrm{D}}^{(i)} a_{i}^{\mathrm{n} \sharp}
$$

Notice that only the dissipative currents and quasicurrents contribute to the entropy production so that the following equation must hold:

$$
\vec{\nabla}_{\vec{r}} \cdot \vec{J}_{\mathrm{R}}^{\varepsilon}=T \vec{\nabla}_{\vec{r}} \cdot \vec{J}_{\mathrm{R}}^{\sigma}+a_{i}^{\mathrm{c} \natural} \vec{\nabla}_{\vec{r}} \cdot \vec{J}_{\mathrm{R}}^{(i)}+a_{i}^{\mathrm{n} \natural} \Phi_{\mathrm{R}}^{(i)} \cdot
$$

Combining equations (21) and (26) now leads to the desired microscopic expression for the dissipation function:

$$
2 \mathfrak{r}(\vec{r}, t)=-\vec{J}_{\mathrm{D}}^{\sigma} \cdot \vec{\nabla}_{\vec{r}} T-\vec{J}_{\mathrm{D}}^{(i)} \cdot \vec{\nabla}_{\vec{r}} a_{i}^{\mathrm{c} \sharp}+\Phi_{\mathrm{D}}^{(i)} a_{i}^{\mathrm{n} \sharp} .
$$

Notice that equations (26) and (28) are in accordance with equation (21). Since the dissipation function $\mathfrak{r}(\vec{r}, t)$ has to be positive semi-definite, equation (28) implies that the dissipative currents and quasicurrents are functions of the thermodynamic forces (18). In fact, these functions are assumed to be linear and homogeneous [31]. With this assumption, it is obvious that the dissipative currents $\vec{J}_{\mathrm{D}}^{(i)}(\vec{r}, t)$ and quasicurrents $\Phi_{\mathrm{D}}^{(i)}(\vec{r}, t)$ corresponding to the conserved relevant variables $a_{i}^{\mathrm{c}}(\vec{r}, t)$ and the non-conserved relevant variables $a_{i}^{\mathrm{n}}(\vec{r}, t)$, respectively, can be derived from a given dissipation functional $\mathfrak{R}$ using equations (19). Analogously, the dissipative entropy current $\overrightarrow{J_{\mathrm{D}}^{\sigma}}(\vec{r}, t)$ can be derived:

$$
\sigma^{\natural}=T, \quad \vec{\sigma}^{\sharp}=-\vec{\nabla}_{\vec{r}} T, \quad \vec{J}_{\mathrm{D}}^{\sigma}=\frac{\delta \Re}{\delta \vec{\sigma}^{\sharp}} .
$$

The dissipative energy current $\overrightarrow{J_{\mathrm{D}}^{\varepsilon}}(\vec{r}, t)$ follows then from equation (25).

Equation (16) together with equations (20) and (25)-(28) embody a microscopic expression for the dynamical equation of the entropy density. This microscopic equation expresses the entropy quasicurrent and the dissipation functional in terms of the (microscopic) currents and quasicurrents of the other relevant variables and constitutes the first main result of this paper. It is noteworthy that the equations in this subsection closely resemble the corresponding hydrodynamic equations [31].

\footnotetext{
${ }^{14}$ Notice that the thermodynamic conjugate of the entropy density is the local absolute temperature: $\sigma^{\natural}(\vec{r}, t)=$ $\partial \varepsilon(\vec{r}, t) / \partial \sigma(\vec{r}, t)=T(\vec{r}, t)$.

15 Throughout the whole paper, a dot ${ }^{\prime}$ denotes a derivation with respect to time $t$. This applies also to time derivatives of correlation functions with two time variables $t$ and $t^{\prime}$ further below, where the dot always means a derivation with respect to $t$ and not to $t^{\prime}$.
} 


\subsection{Equilibrium correlations}

We now consider the dynamics of the entropy time correlation function $C_{\sigma \sigma}\left(\vec{r}, \vec{r}^{\prime}, t, t^{\prime}\right)$. In the linear regime near equilibrium, the equilibrium time correlation function (Kubo function [8]) of two variables $\hat{X}(\vec{r}, t)$ and $\hat{Y}(\vec{r}, t)$ is defined as $[8,11]$

$$
C_{X Y}\left(\vec{r}, \vec{r}^{\prime}, t, t^{\prime}\right)=\left\langle\Delta \hat{X}^{\mathrm{eq}}(\vec{r}, t) \mid \Delta \hat{Y}^{\mathrm{eq}}\left(\vec{r}^{\prime}, t^{\prime}\right)\right\rangle_{\mathrm{eq}} .
$$

Here, the letters 'eq' denote equilibrium quantities, $\langle\cdot \mid \cdot\rangle_{\mathrm{eq}}$ is Mori's scalar product [11] and $\Delta \hat{X}^{\mathrm{eq}}(\vec{r}, t)=\hat{X}(\vec{r}, t)-X^{\mathrm{eq}}(\vec{r})$ with $X^{\mathrm{eq}}(\vec{r})=\langle\hat{X}(\vec{r}, t)\rangle_{\mathrm{eq}}$ are the equilibrium fluctuations of the variable $\hat{X}(\vec{r}, t)$. Since they are associated with equilibrium fluctuations, the time correlation functions (30) are translationally invariant with respect to time: $C_{X Y}\left(\vec{r}, \vec{r}^{\prime}, t, t^{\prime}\right)=$ $C_{X Y}\left(\vec{r}, \vec{r}^{\prime}, t-t^{\prime}\right)$. For the derivation of the dynamics of $C_{\sigma \sigma}\left(\vec{r}, \vec{r}^{\prime}, t, t^{\prime}\right)$, we need an expression for the operator $\hat{\sigma}(\vec{r}, t)$ of the entropy density, but such an operator is not known in general. Therefore, we assume that the local formulation of the first law of thermodynamics (22) also holds for the corresponding operators $[6,8,12,36,37]$. This leads to the equation ${ }^{16}$

$$
\mathrm{d} \hat{\varepsilon}=T \mathrm{~d} \hat{\sigma}+a_{i}^{\natural} \mathrm{d} \hat{a}_{i}
$$

that expresses the entropy density operator $\hat{\sigma}(\vec{r}, t)$ in terms of the internal energy density operator $\hat{\varepsilon}(\vec{r}, t)$ and the operators $\hat{a}_{i}(\vec{r}, t)$ for the other relevant variables that are known. Notice that the relevant variables $\hat{a}_{i} \in\left\{\hat{a}_{i}^{\mathrm{c}}, \hat{a}_{i}^{\mathrm{n}}\right\}$ are not distinguished into conserved and non-conserved variables in this paragraph. Equation (31) can now be rearranged into

$$
\dot{\hat{\sigma}}=\frac{1}{T} \dot{\hat{\varepsilon}}-\frac{a_{i}^{\natural}}{T} \dot{\hat{a}}_{i}
$$

providing a dynamical equation for the entropy density operator. Using the definition (30) of the time correlation functions and their symmetry properties described in [8], relations between the time-derived correlation functions $\dot{C}_{X Y}\left(\vec{r}, \vec{r}^{\prime}, t, t^{\prime}\right)$ with $X, Y \in\left\{\sigma, \varepsilon, a_{i}\right\}$ can be derived. A combination of these relations leads to the following transport equation for the entropy time correlation function:

$$
\begin{aligned}
\dot{C}_{\sigma \sigma}\left(\vec{r}, \vec{r}^{\prime}, t, t^{\prime}\right)= & \frac{1}{T(\vec{r}, t) T\left(\vec{r}^{\prime}, t^{\prime}\right)} \dot{C}_{\varepsilon \varepsilon}\left(\vec{r}, \vec{r}^{\prime}, t, t^{\prime}\right)-\frac{a_{i}^{\natural}(\vec{r}, t)}{T(\vec{r}, t) T\left(\vec{r}^{\prime}, t^{\prime}\right)} \dot{C}_{a_{i} \varepsilon}\left(\vec{r}, \vec{r}^{\prime}, t, t^{\prime}\right) \\
& -\frac{a_{i}^{\natural}\left(\vec{r}^{\prime}, t^{\prime}\right)}{T(\vec{r}, t) T\left(\vec{r}^{\prime}, t^{\prime}\right)} \dot{C}_{\varepsilon a_{i}}\left(\vec{r}, \vec{r}^{\prime}, t, t^{\prime}\right)+\frac{a_{i}^{\natural}(\vec{r}, t) a_{j}^{\natural}\left(\vec{r}^{\prime}, t^{\prime}\right)}{T(\vec{r}, t) T\left(\vec{r}^{\prime}, t^{\prime}\right)} \dot{C}_{a_{i} a_{j}}\left(\vec{r}, \vec{r}^{\prime}, t, t^{\prime}\right) .
\end{aligned}
$$

This equation constitutes the second main result of this paper, since it brings about a microscopic expression for the entropy time correlation function.

\subsection{Dissipation}

By the entropy balance equation (16) with the quasicurrent (20) and the entropy production (21), the dissipation function (28) is directly associated with dissipation. Also, the time-derived correlation functions in equation (33) can be related to dissipation, when the validity of the fluctuation-dissipation theorem $[8,38]$ is assumed. For systems sufficiently close to thermodynamic equilibrium, the fluctuation-dissipation theorem implies the relation

$$
\dot{C}_{a_{i} a_{j}}\left(\vec{r}, \vec{r}^{\prime}, t, t^{\prime}\right)=\frac{2}{\mathrm{i} \beta} \chi_{i j}^{\prime \prime}\left(\vec{r}, \vec{r}^{\prime}, t, t^{\prime}\right)
$$

\footnotetext{
${ }^{16}$ Although equation (31) provides an expression for the entropy operator $\hat{\sigma}(\vec{r}, t)$, this variable cannot be incorporated into EDDFT in the usual way, since the MZFT requires a set of independent relevant variables [11]. Notice that by averaging equation (31) with $\operatorname{Tr}(\rho(t) \cdot)$, one obtains the local formulation of the first law of thermodynamics (22).
} 
or equivalently in Fourier space (see appendix A.1)

$$
\widetilde{\chi}_{i j}^{\prime \prime}\left(\vec{r}, \vec{r}^{\prime}, \omega\right)=\frac{\beta}{2} \omega \widetilde{C}_{a_{i} a_{j}}\left(\vec{r}, \vec{r}^{\prime}, \omega\right)
$$

between the time correlation function $C_{a_{i} a_{j}}\left(\vec{r}, \vec{r}^{\prime}, t, t^{\prime}\right)$ and the absorptive response function ${ }^{17}[11]$

$$
\chi_{i j}^{\prime \prime}\left(\vec{r}, \vec{r}^{\prime}, t, t^{\prime}\right)=\frac{1}{2 \hbar}\left\langle\left[\hat{a}_{i}(\vec{r}, t), \hat{a}_{j}\left(\vec{r}^{\prime}, t^{\prime}\right)\right]\right\rangle_{\mathrm{eq}}
$$

for any variables $\hat{a}_{i}(\vec{r}, t)$ and $\hat{a}_{j}(\vec{r}, t)$ also including the internal energy density $\hat{\varepsilon}(\vec{r}, t)$ and the entropy density $\hat{\sigma}(\vec{r}, t)$. In the following, the meaning of the dissipation functional $\mathfrak{R}$ and the absorptive response function $\chi_{i j}^{\prime \prime}\left(\vec{r}, \vec{r}^{\prime}, t, t^{\prime}\right)$ in the context of dissipation is considered in more detail.

3.3.1. Nonequilibrium dynamics. The total work $W(t)$ done on a system with the effective Hamiltonian $\hat{H}_{\text {eff }}(t)$ is given by [8, 39]

$$
W(t)=\operatorname{Tr}\left(\rho(t) \hat{H}_{\mathrm{eff}}(t)\right) .
$$

Its rate of change $\dot{W}(t)$ is the energy dissipated per time in this system and identical with the dissipation functional $[8,12]$

$$
\mathfrak{R}=\dot{W}(t)=\operatorname{Tr}\left(\rho(t) \dot{\hat{H}}_{\mathrm{eff}}(t)\right)=-\sum_{i=1}^{n} \int_{\mathbb{R}^{3}} \mathrm{~d}^{3} r a_{i}(\vec{r}, t) \dot{a}_{i}^{\natural}(\vec{r}, t),
$$

where the averaged relevant variables $a_{i}(\vec{r}, t)$ now also include the internal energy density $\varepsilon(\vec{r}, t)$, but not the dependent entropy density $\sigma(\vec{r}, t)$. Notice that equation (38) can be understood as a generalization of equation (33) in [16]. The time integral of equation (38) is the total amount of dissipated energy

$$
W_{\mathrm{diss}}=\int_{\mathbb{R}} \mathrm{d} t \Re=\sum_{i=1}^{n} \int_{\mathbb{R}} \mathrm{d} t \int_{\mathbb{R}^{3}} \mathrm{~d}^{3} r \dot{a}_{i}(\vec{r}, t) a_{i}^{\natural}(\vec{r}, t) .
$$

3.3.2. Equilibrium correlations. Using the fluctuation-dissipation theorem (34), the dissipated energy (39) can also be expressed in dependence of the time-derived correlation functions $\dot{C}_{a_{i} a_{j}}\left(\vec{r}, \vec{r}^{\prime}, t, t^{\prime}\right)$ or equivalently in dependence of the absorptive response functions $\chi_{i j}^{\prime \prime}\left(\vec{r}, \vec{r}^{\prime}, t, t^{\prime}\right)$. For this purpose, the symmetric equilibrium susceptibility matrix (complex response function) [11]

$$
\chi_{i j}\left(\vec{r}, \vec{r}^{\prime}, t, t^{\prime}\right)=\left.\frac{\delta a_{i}(\vec{r}, t)}{\delta a_{j}^{\natural}\left(\vec{r}^{\prime}, t^{\prime}\right)}\right|_{\mathrm{eq}}
$$

is used in order to express the averaged relevant variables $a_{i}(\vec{r}, t)=a_{i}^{\mathrm{eq}}(\vec{r})+\Delta a_{i}(\vec{r}, t)$ in terms of the corresponding thermodynamic conjugates $a_{i}^{\natural}(\vec{r}, t)^{18}$ :

$$
\Delta a_{i}(\vec{r}, t)=\sum_{j=1}^{n} \int_{\mathbb{R}} \mathrm{d} t^{\prime} \int_{\mathbb{R}^{3}} \mathrm{~d}^{3} r^{\prime} \chi_{i j}\left(\vec{r}, \vec{r}^{\prime}, t, t^{\prime}\right) a_{j}^{\natural}\left(\vec{r}^{\prime}, t^{\prime}\right) .
$$

\footnotetext{
${ }^{17}$ See $[8,12,39]$ for the symmetry properties of the absorptive response function $\chi_{i j}^{\prime \prime}\left(\vec{r}, \vec{r}^{\prime}, t, t^{\prime}\right)$.

${ }^{18}$ In the linear regime near equilibrium, equation (41) follows directly from a functional Taylor expansion of the variable $a_{i}(\vec{r}, t)$ with respect to the thermodynamic conjugates $a_{i}^{\natural}(\vec{r}, t)$ about the equilibrium state, in which the thermodynamic conjugates vanish.
} 
Inserting this expression into equation (39) leads to

$$
W_{\mathrm{diss}}=\sum_{i, j=1}^{n} \int_{\mathbb{R}} \mathrm{d} t \int_{\mathbb{R}} \mathrm{d} t^{\prime} \int_{\mathbb{R}^{3}} \mathrm{~d}^{3} r \int_{\mathbb{R}^{3}} \mathrm{~d}^{3} r^{\prime} a_{i}^{\natural}(\vec{r}, t) \dot{\chi}_{i j}\left(\vec{r}, \vec{r}^{\prime}, t, t^{\prime}\right) a_{j}^{\natural}\left(\vec{r}^{\prime}, t^{\prime}\right) .
$$

In Fourier space, the complex response function (40) can be decomposed as [8, 11]

$$
\tilde{\chi}_{i j}\left(\vec{r}, \vec{r}^{\prime}, \omega\right)=\tilde{\chi}_{i j}^{\prime}\left(\vec{r}, \vec{r}^{\prime}, \omega\right)+\mathrm{i} \tilde{\chi}_{i j}^{\prime \prime}\left(\vec{r}, \vec{r}^{\prime}, \omega\right)
$$

with the (Fourier transformed) reactive response function $\tilde{\chi}_{i j}^{\prime}\left(\vec{r}, \vec{r}^{\prime}, \omega\right)$ and absorptive response function $\tilde{\chi}_{i j}^{\prime \prime}\left(\vec{r}, \vec{r}^{\prime}, \omega\right)$. Using this decomposition, together with the symmetry properties $[8,12,39] \widetilde{\chi}_{j i}^{\prime}\left(\vec{r}^{\prime}, \vec{r},-\omega\right)=\widetilde{\chi}_{i j}^{\prime}\left(\vec{r}, \vec{r}^{\prime}, \omega\right)$ and $-\tilde{\chi}_{j i}^{\prime \prime}\left(\vec{r}^{\prime}, \vec{r},-\omega\right)=\tilde{\chi}_{i j}^{\prime \prime}\left(\vec{r}, \vec{r}^{\prime}, \omega\right)$ of the reactive and absorptive response functions, respectively, as well as Parseval's theorem (see appendix A.2), it can be shown that equation (42) is equivalent to

$$
W_{\mathrm{diss}}=\frac{1}{2 \pi} \sum_{i, j=1}^{n} \int_{\mathbb{R}^{3}} \mathrm{~d}^{3} r \int_{\mathbb{R}^{3}} \mathrm{~d}^{3} r^{\prime} \int_{\mathbb{R}} \mathrm{d} \omega \widetilde{a}_{i}^{\natural}(\vec{r},-\omega) \omega \widetilde{\chi}_{i j}^{\prime \prime}\left(\vec{r}, \vec{r}^{\prime}, \omega\right) \widetilde{a}_{j}^{\natural}\left(\vec{r}^{\prime}, \omega\right) .
$$

In accordance with the second law of thermodynamics, the dissipated energy $W_{\text {diss }}$ must be non-negative in a stable system (see section 2.3 ). Since this stability condition must also hold for any generalized Helmholtz free-energy functional $\mathcal{F}$ and hence for an arbitrary set of thermodynamic conjugates $\widetilde{a}_{i}^{\natural}(\vec{r}, \omega)$, the product $\omega \tilde{\chi}_{i j}^{\prime \prime}\left(\vec{r}, \vec{r}^{\prime}, \omega\right){ }^{19}$ has to be positive semidefinite for any $\omega$ [8]. As a consequence of equation (35), $\widetilde{C}_{a_{i} a_{j}}\left(\vec{r}, \vec{r}^{\prime}, \omega\right)$ also has to be positive semi-definite.

\section{Extended dynamical density functional theory}

So far, a general set of relevant conserved or non-conserved variables $\hat{a}_{i}(\vec{r}, t)$ with general transport equations (7) and (9), respectively, has been considered. In this section, we now focus on current EDDFT [11] with its conserved, real, and independent variables $\hat{a}_{i}(\vec{r}, t) \equiv \hat{a}_{i}^{\mathrm{c}}(\vec{r}, t)$ with $i \in\{1, \ldots, n\}$ including the internal energy density and with the corresponding specified transport equations. We first summarize the EDDFT equations and the associated transport equations for time correlation functions. Afterwards, we discuss the derivation of EDDFT from a dissipation functional and consider the hydrodynamic limit of EDDFT. These considerations are valid for general variables $\hat{a}_{i}(\vec{r}, t)$ that have to be conserved, real, and independent, as usual in the context of EDDFT [11]. For more particular EDDFT equations with the concentrations $\hat{c}_{i}(\vec{r}, t)$ and the energy density $\hat{\varepsilon}(\vec{r}, t)$ as relevant variables, see [11].

\subsection{Nonequilibrium dynamics}

In its currently most general form, the EDDFT equations are given by [11]

$$
\dot{a}_{i}(\vec{r}, t)+\vec{\nabla}_{\vec{r}} \cdot \vec{J}^{a_{i}}(\vec{r}, t)=0
$$

with the currents $\vec{J}^{a_{i}}(\vec{r}, t)=\vec{J}_{\mathrm{R}}^{u_{i}}(\vec{r}, t)+\vec{J}_{\mathrm{D}}^{a_{i}}(\vec{r}, t)$ and

$$
\begin{aligned}
& \vec{J}_{\mathrm{R}}^{a_{i}}(\vec{r}, t)=\operatorname{Tr}\left(\rho(t) \hat{\vec{J}^{(i)}}(\vec{r}, 0)\right), \\
& \vec{J}_{\mathrm{D}}^{a_{i}}(\vec{r}, t)=-\sum_{j=1}^{n} \int_{\mathbb{R}^{3}} \mathrm{~d}^{3} r^{\prime} \beta D^{(i j)}\left(\vec{r}, \vec{r}^{\prime}, t\right) \vec{\nabla}_{\vec{r}^{\prime}} a_{j}^{\natural}\left(\vec{r}^{\prime}, t\right) .
\end{aligned}
$$

Here, $D^{(i j)}\left(\vec{r}, \vec{r}^{\prime}, t\right)$ denotes a diffusion tensor, for which explicit expressions can be found in [11]. We note that $D^{(i j)}\left(\vec{r}, \vec{r}^{\prime}, t\right)$ in equation (47) contains all non-instantaneous contributions. These are mainly dissipative in nature. For certain systems such as, for example, nematic liquid crystals, there are also reversible contributions even in the hydrodynamic regime $[6,8]$.

${ }^{19}$ Notice that the sign of this expression depends on the definition of the Fourier transformation. 


\subsection{Equilibrium correlations}

In the linear regime near equilibrium, the corresponding transport equations for the equilibrium time correlation functions $C_{a_{i} a_{j}}\left(\vec{r}, \vec{r}^{\prime}, t\right)^{20}$ are given by [11]

$$
\dot{C}_{a_{i} a_{j}}\left(\vec{r}, \vec{r}^{\prime}, t\right)+\vec{\nabla}_{\vec{r}} \cdot \mathrm{J}^{(i j)}\left(\vec{r}, \vec{r}^{\prime}, t\right)=0
$$

with the total currents $\mathrm{J}^{(i j)}\left(\vec{r}, \vec{r}^{\prime}, t\right)=\mathrm{J}_{\mathrm{R}}^{(i j)}\left(\vec{r}, \vec{r}^{\prime}, t\right)+\mathrm{J}_{\mathrm{D}}^{(i j)}\left(\vec{r}, \vec{r}^{\prime}, t\right)$ and their contributions

$$
\begin{aligned}
& \mathrm{J}_{\mathrm{R}}^{(i j)}\left(\vec{r}, \vec{r}^{\prime}, t\right)=-\sum_{k=1}^{n} \int_{\mathbb{R}^{3}} \mathrm{~d}^{3} r^{\prime \prime} \Omega_{\mathrm{eq}}^{(i k)}\left(\vec{r}, \vec{r}^{\prime \prime}\right) C_{k j}\left(\vec{r}^{\prime \prime}, \vec{r}^{\prime}, t\right), \\
& \mathrm{J}_{\mathrm{D}}^{(i j)}\left(\vec{r}, \vec{r}^{\prime}, t\right)=-\sum_{k=1}^{n} \int_{\mathbb{R}^{3}} \mathrm{~d}^{3} r^{\prime \prime} \Gamma_{\mathrm{eq}}^{(i k)}\left(\vec{r}, \vec{r}^{\prime \prime}\right) C_{k j}\left(\vec{r}^{\prime \prime}, \vec{r}^{\prime}, t\right) .
\end{aligned}
$$

For explicit expressions for $\Omega_{\mathrm{eq}}^{(i j)}\left(\vec{r}, \vec{r}^{\prime}\right)$ and $\Gamma_{\mathrm{eq}}^{(i j)}\left(\vec{r}, \vec{r}^{\prime}\right)$, see [11].

\subsection{Dissipation}

The reversible currents (46) of EDDFT often vanish. This is generally the case when all relevant variables $a_{i}(\vec{r}, t)$ have the same time-reversal behavior [8]. Then only the dissipative currents (47) remain and the complete EDDFT equations (45) can be derived from a dissipation functional that is defined by equations (17) and (28). Notice that the internal energy density is treated separately from the other relevant variables in equation (28), while it is included in the relevant variables $a_{i}(\vec{r}, t)$ in equations (45)-(47). For instance, the traditional DDFT of Marconi and Tarazona [13-15]

$$
\begin{aligned}
& \dot{\rho}(\vec{r}, t)+\vec{\nabla}_{\vec{r}} \cdot \vec{J}^{\rho}(\vec{r}, t)=0, \\
& \overrightarrow{J^{\rho}}(\vec{r}, t)=-\beta D_{0} \rho(\vec{r}, t) \vec{\nabla}_{\vec{r}} \rho^{\natural}(\vec{r}, t)
\end{aligned}
$$

with the one-particle density $\rho(\vec{r}, t)$ and the translational short-time diffusion coefficient $D_{0}$ can be derived formally from the dissipation functional $\mathfrak{R}$ with the dissipation function

$$
\mathfrak{r}_{\mathrm{MT}}(\vec{r}, t)=\frac{1}{2} \beta D_{0} \rho(\vec{r}, t)\left(\vec{\nabla}_{\vec{r}} \rho^{\natural}(\vec{r}, t)\right)^{2} .
$$

This reformulation of DDFT in terms of a dissipation functional constitutes an alternative representation of DDFT besides the usual DDFT equations. Such a representation by a dissipation functional is very advantageous when some of the relevant variables (in this example the one-particle density $\rho(\vec{r}, t))$ have to be parametrized by other order-parameter fields and dynamical equations for these parameterizing order-parameter fields are needed. The derivation of PFC models from DDFT, for example, involves such a parametrization of the one-particle density. While this is pretty straightforward for isotropic systems with a purely translational particle density [40, 41], the derivation of the dynamical equations becomes considerably more complicated for additional orientational degrees of freedom both in two $[19,20,26]$ and three [18] spatial dimensions (for a recent review see [21]). The use of a dissipation functional instead of a DDFT equation considerably simplifies the situation.

\subsection{Special cases of the EDDFT equations}

In this subsection, we consider two special cases of the EDDFT equations (45)-(47) in order to demonstrate their applicability and to compare them to equations that are known from the literature. These special cases are the dynamical equations for non-isothermal colloidal suspensions and the hydrodynamic limit of the EDDFT equations.

${ }^{20}$ Here, we made use of the fact that the equilibrium time correlation functions $C_{a_{i} a_{j}}\left(\vec{r}, \vec{r}^{\prime}, t, t^{\prime}\right)$ are translationally invariant with respect to time so that $t^{\prime}$ can be omitted. 
4.4.1. Non-isothermal colloidal suspensions. We consider a non-isothermal colloidal suspension, whose state is described by a given generalized Helmholtz free-energy functional $\mathcal{F}[c, T]$ in terms of two relevant variables, the (conserved) concentration field $c(\vec{r}, t)$ and the (non-conserved) temperature field $T(\vec{r}, t)$. Such a functional can, for example, be obtained from static classical DFT [42-44], when a local approximation is applied to the DFT functional $\mathcal{F}(T,[c])$ with the spatially homogeneous temperature parameter $T$. Instead of the temperature field $T(\vec{r}, t)$, the internal energy density $\varepsilon(\vec{r}, t)$ or the entropy density $\sigma(\vec{r}, t)$ lead-in combination with the concentration field $c(\vec{r}, t)$ - to a set of two relevant variables that are appropriate for describing the considered system (see appendix B for explicit expressions for the Hamiltonian and the relevant variables). Here, we choose $c(\vec{r}, t)$ and $\sigma(\vec{r}, t)$ as the relevant variables and the generalized internal energy functional

$$
\mathcal{E}[c, \sigma]=\mathcal{F}[c, T]+\int_{\mathbb{R}^{3}} \mathrm{~d}^{3} r T(\vec{r}, t) \sigma(\vec{r}, t)
$$

as the corresponding thermodynamic functional. The thermodynamic conjugates of the relevant variables are thus defined as $c^{\natural}(\vec{r}, t)=\delta \mathcal{E} / \delta c(\vec{r}, t)=\delta \mathcal{F} / \delta c(\vec{r}, t)$ and $\sigma^{\natural}(\vec{r}, t)=$ $\delta \mathcal{E} / \delta \sigma(\vec{r}, t)=T(\vec{r}, t)$ in this section. Since $c(\vec{r}, t), \varepsilon(\vec{r}, t)$ and $\sigma(\vec{r}, t)$ are even under parity inversion and time reversal, there are no reversible currents [11]: $\overrightarrow{J_{\mathrm{R}}^{c}}(\vec{r}, t)=\overrightarrow{J_{\mathrm{R}}^{\varepsilon}}(\vec{r}, t)=$ $\overrightarrow{J_{\mathrm{R}}^{\sigma}}(\vec{r}, t)=\overrightarrow{0}$. Hence, the dynamics of such a non-isothermal colloidal suspension is described by

$$
\begin{aligned}
& \dot{c}(\vec{r}, t)+\vec{\nabla}_{\vec{r}} \cdot \vec{J}_{\mathrm{D}}^{c}(\vec{r}, t)=0, \\
& \dot{\sigma}(\vec{r}, t)+\vec{\nabla}_{\vec{r}} \cdot \vec{J}_{\mathrm{D}}^{\sigma}(\vec{r}, t)=Q^{\sigma}(\vec{r}, t) .
\end{aligned}
$$

These dynamical equations follow directly from equations (45), (16), and (20). The dissipative currents in equations (55) and (56) result from equation (47) and are given by

$\overrightarrow{J_{\mathrm{D}}^{c}}(\vec{r}, t)=-\int_{\mathbb{R}^{3}} \mathrm{~d}^{3} r^{\prime} \beta D^{(c c)}\left(\vec{r}, \vec{r}^{\prime}, t\right) \vec{\nabla}_{\vec{r}^{\prime}} c^{\natural}\left(\vec{r}^{\prime}, t\right)-\int_{\mathbb{R}^{3}} \mathrm{~d}^{3} r^{\prime} \beta D^{(c \sigma)}\left(\vec{r}, \vec{r}^{\prime}, t\right) \vec{\nabla}_{\vec{r}^{\prime}} \sigma^{\natural}\left(\vec{r}^{\prime}, t\right)$,

$\overrightarrow{J_{\mathrm{D}}^{\sigma}}(\vec{r}, t)=-\int_{\mathbb{R}^{3}} \mathrm{~d}^{3} r^{\prime} \beta D^{(\sigma c)}\left(\vec{r}, \vec{r}^{\prime}, t\right) \vec{\nabla}_{\vec{r}^{\prime}} c^{\natural}\left(\vec{r}^{\prime}, t\right)-\int_{\mathbb{R}^{3}} \mathrm{~d}^{3} r^{\prime} \beta D^{(\sigma \sigma)}\left(\vec{r}, \vec{r}^{\prime}, t\right) \vec{\nabla}_{\vec{r}^{\prime}} \sigma^{\natural}\left(\vec{r}^{\prime}, t\right)$.

Using these expressions for the dissipative currents $\overrightarrow{J_{\mathrm{D}}^{c}}(\vec{r}, t)$ and $\overrightarrow{J_{\mathrm{D}}^{\sigma}}(\vec{r}, t)$, an explicit equation for the dissipative internal energy current $\overrightarrow{J_{\mathrm{D}}^{\varepsilon}}(\vec{r}, t)$ can be derived from equation (25). This explicit equation is given by

$\overrightarrow{J_{\mathrm{D}}^{\varepsilon}}(\vec{r}, t)=-\int_{\mathbb{R}^{3}} \mathrm{~d}^{3} r^{\prime} \beta D^{(\varepsilon c)}\left(\vec{r}, \vec{r}^{\prime}, t\right) \vec{\nabla}_{\vec{r}^{\prime}} c^{\natural}\left(\vec{r}^{\prime}, t\right)-\int_{\mathbb{R}^{3}} \mathrm{~d}^{3} r^{\prime} \beta D^{(\varepsilon \sigma)}\left(\vec{r}, \vec{r}^{\prime}, t\right) \vec{\nabla}_{\vec{r}^{\prime}} \sigma^{\natural}\left(\vec{r}^{\prime}, t\right)$

with the diffusion tensors

$$
\begin{aligned}
& D^{(\varepsilon c)}\left(\vec{r}, \vec{r}^{\prime}, t\right)=c^{\natural}(\vec{r}, t) D^{(c c)}\left(\vec{r}, \vec{r}^{\prime}, t\right)+\sigma^{\natural}(\vec{r}, t) D^{(\sigma c)}\left(\vec{r}, \vec{r}^{\prime}, t\right), \\
& D^{(\varepsilon \sigma)}\left(\vec{r}, \vec{r}^{\prime}, t\right)=c^{\natural}(\vec{r}, t) D^{(c \sigma)}\left(\vec{r}, \vec{r}^{\prime}, t\right)+\sigma^{\natural}(\vec{r}, t) D^{(\sigma \sigma)}\left(\vec{r}, \vec{r}^{\prime}, t\right) .
\end{aligned}
$$

4.4.2. Hydrodynamic limit. All expressions presented in this paper so far are beyond the scope of hydrodynamics and are also applicable for non-hydrodynamic wave vectors $\vec{k}$ and frequencies $\omega$. In the hydrodynamic limit $(\vec{k} \rightarrow \overrightarrow{0}, \omega \rightarrow 0)$, the transport matrices become constant and the transport equations simplify considerably. In particular, the diffusion tensor $D^{(i j)}\left(\vec{r}, \vec{r}^{\prime}, t\right)$ in equation (47) and the transport matrices $\Omega_{\mathrm{eq}}^{(i j)}\left(\vec{r}, \vec{r}^{\prime}\right)$ and $\Gamma_{\mathrm{eq}}^{(i j)}\left(\vec{r}, \vec{r}^{\prime}\right)$ 
in equations (49) and (50), respectively, are constant in the hydrodynamic limit (see [11] for details). For example, equations (55)-(59) reduce to

$$
\begin{aligned}
& \dot{c}(\vec{r}, t)+\vec{\nabla}_{\vec{r}} \cdot \vec{J}_{\mathrm{H}}^{c}(\vec{r}, t)=0, \\
& \dot{\sigma}(\vec{r}, t)+\vec{\nabla}_{\vec{r}} \cdot \vec{J}_{\mathrm{H}}^{\sigma}(\vec{r}, t)=Q_{\mathrm{H}}^{\sigma}(\vec{r}, t), \\
& \dot{\varepsilon}(\vec{r}, t)=\dot{\sigma}(\vec{r}, t) T(\vec{r}, t)+\dot{c}(\vec{r}, t) c^{\natural}(\vec{r}, t)
\end{aligned}
$$

with the entropy production

$$
Q_{\mathrm{H}}^{\sigma}(\vec{r}, t)=\frac{\beta}{T(\vec{r}, t)}\left(D_{c c}\left(\vec{\nabla}_{\vec{r}} c^{\natural}(\vec{r}, t)\right)^{2}+2 D_{c \sigma} \vec{\nabla}_{\vec{r}} c^{\natural}(\vec{r}, t) \cdot \vec{\nabla}_{\vec{r}} T(\vec{r}, t)+D_{\sigma \sigma}\left(\vec{\nabla}_{\vec{r}} T(\vec{r}, t)\right)^{2}\right)
$$

in the hydrodynamic limit. Here, $c^{\natural}(\vec{r}, t)$ denotes the functional derivative $c^{\natural}(\vec{r}, t)=$ $\delta \mathcal{F} / \delta c(\vec{r}, t)$ of the Helmholtz free-energy functional $\mathcal{F}[c, T]$ and $D_{c c}, D_{c \sigma}$, and $D_{\sigma \sigma}$ are systemdependent constants. The currents $\overrightarrow{J_{\mathrm{H}}^{c}}(\vec{r}, t)$ and $\overrightarrow{J_{\mathrm{H}}^{\sigma}}(\vec{r}, t)$ can be obtained from the dissipation functional corresponding to the entropy production (65) by functional differentiation with respect to the thermodynamic forces. This results in

$$
\begin{aligned}
& \vec{J}_{\mathrm{H}}^{c}(\vec{r}, t)=-\beta D_{c c} \vec{\nabla}_{\vec{r}} c^{\natural}(\vec{r}, t)-\beta D_{c \sigma} \vec{\nabla}_{\vec{r}} T(\vec{r}, t), \\
& \vec{J}_{\mathrm{H}}^{\sigma}(\vec{r}, t)=-\beta D_{c \sigma} \vec{\nabla}_{\vec{r}} c^{\natural}(\vec{r}, t)-\beta D_{\sigma \sigma} \vec{\nabla}_{\vec{r}} T(\vec{r}, t) .
\end{aligned}
$$

\section{Conclusions and perspectives}

For a general set of independent relevant variables, we derived microscopic transport equations for the entropy density (see equations (16), (20), (25), and (26)) as well as for the entropy time correlation function (see equation (33)) in the framework of linear irreversible thermodynamics. We thus complemented current EDDFT [11] with a balance equation for the entropy density that has previously not yet been considered in the context of EDDFT as a relevant variable. We furthermore derived the microscopic expression (28) defining a dissipation functional, from which the dissipative dynamics of EDDFT can be derived. This reformulation of EDDFT in terms of a dissipation functional complements the EDDFT equations and also states that DDFT [13-17] can be derived from a dissipation functional. Our dissipation functional is especially useful, if some of the relevant variables have to be parametrized and dynamical equations for the parameterizing quantities are needed. This is particularly the case in the derivation of PFC models [18-21] from EDDFT.

Our theory can be applied by approximating the diffusion tensor in equation (47) and the transport matrices in equations (49) and (50) by the corresponding expressions in the hydrodynamic limit (see section 4.4.2). The latter involve only a few transport coefficients that can be adjusted to actual simulation data for a given system. Then the resulting equations are in principle closed and can be applied to describe a variety of relaxation processes. One prominent example is the crystallization process from an undercooled melt where latent heat is produced such that there is a coupling between the density field and the entropy density. Latent heat production yields a hindering of crystal growth and is relevant both for molecular systems and for dusty plasmas. For future perspectives, the application of our theoretical framework towards crystallization processes is planned.

It is possible to generalize our approach to include macroscopic variables as well. These are variables that relax on a sufficiently long but finite time scale in the long-wavelength limit. Their combination with the hydrodynamic approach has been pioneered by Khalatnikov 
near the $\lambda$ transition in superfluid ${ }^{4} \mathrm{He}$ [45]. In this case, the degree of order, which is the modulus of the macroscopic wave function, becomes a slow macroscopic variable as this second-order phase transition is approached. The $\lambda$ transition is, in fact, the second-order phase transition, which has been studied more quantitatively than any other phase transition, both theoretically [46-49] and experimentally [50, 51] testing universality concepts as well as deviations from these concepts in detail. Quantitative agreement was found between theory and experiment. Later, the degree of order has also been introduced as a macroscopic variable near other phase transitions of second or weakly first order. Examples include phase transitions in liquid crystals such as the isotropic-nematic phase transition [52], the nematic-smectic A phase transition [53], the uniaxial-biaxial nematic phase transition [54-56], and the nematiccolumnar phase transition [57] as well as the normal fluid-superfluid ${ }^{3} \mathrm{He}-\mathrm{A}$ phase transition in ${ }^{3} \mathrm{He}[58]$.

Another class of slow variables arises when there is an energy scale in the system which is much smaller than all other energy scales. A classical example of this case is superfluid ${ }^{3} \mathrm{He}-\mathrm{A}$, where the magnetic dipole-dipole interaction is of order $2 \times 10^{-7} \mathrm{~K}$ (temperature associated with the appropriate energy) and thus very small compared to all other energy scales including the energy gap. As a consequence of the presence of the magnetic dipole-dipole interaction the magnetization density is no longer a truly conserved hydrodynamic variable, but a rather long-lived macroscopic variable, whose dynamics can be incorporated into the hydrodynamics of superfluid ${ }^{3} \mathrm{He}-\mathrm{A}[37,59]$, where it turns out to be crucial for the understanding of the NMR spectra in the superfluid phase.

A class of macroscopic variables, which is particularly important for complex fluids including polymers, colloids, liquid crystalline elastomers etc. are strain fields associated with a transient network. In these cases the system reacts like a fluid below a certain frequency, the so-called Maxwell frequency, and like a solid or glass above this frequency. Therefore, the consequences of a transient strain field on the macroscopic behavior by combining it with the hydrodynamic approach have also been explored [60-65]. It therefore seems a natural next step to incorporate macroscopic variables such as a transient strain field into the EDDFT approach.

In contrast to hydrodynamic equations, our results are not restricted to the hydrodynamic regime $(\vec{k} \rightarrow \overrightarrow{0}, \omega \rightarrow 0)$, but are also applicable for larger wave vectors $\vec{k}$ and frequencies $\omega$. However, our results based on linear irreversible thermodynamics are restricted to systems that are sufficiently close to thermodynamic equilibrium, where the local formulations of the first and second law of thermodynamics are valid. This is generally the case for all passive systems, but not for active systems. The generalization of our results to active systems that are far from thermodynamic equilibrium, where the entropy production can also be negative locally or in a subsystem and linear irreversible thermodynamics is not applicable, will therefore be an important task for the future. In this context, the crucial question for the existence of an entropy density operator in particular will have to be answered.

Entropy has been considered in the context of DDFT in recent work by Anero et al [66] and Schmidt [67]. In our work, described in the present paper, a generalized Helmholtz freeenergy functional is chosen as an appropriate thermodynamic functional, the DDFT equations in the work of Anero et al are based on an entropy functional and do not include the entropy density as a thermodynamic variable [66]. As a further difference, the balance equation for the entropy density and the dissipation functional are not used in the approach of Anero et al. Schmidt, on the other hand, proposed DDFT equations for a one-particle density and an internal energy density on the basis of a generalized grand-canonical potential functional that depends functionally on the one-particle density and the entropy density and is minimized by both densities in thermodynamic equilibrium [67]. 


\section{Acknowledgments}

We thank Pep Español for helpful discussions. This work was financially supported by the German Research Foundation (DFG) within SPP 1296 and by the European Research Council (ERC Advanced Grant INTERCOCOS, grant agreement 267499). RW gratefully acknowledges financial support from a Postdoctoral Research Fellowship (grant no. WI 4170/1-1) of the DFG.

\section{Appendix A. Fourier transformation}

Since there are different definitions of the Fourier transformation in the literature, here we give the definition we used in the context of the work presented. We further state a useful theorem related to the Fourier transformation.

\section{A.1. Definition}

Within the definition we used, the Fourier transformation of a time-dependent function $X(t)$ is given by

$$
\tilde{X}(\omega)=\int_{\mathbb{R}} \mathrm{d} t X(t) \mathrm{e}^{\mathrm{i} \omega t}, \quad X(t)=\frac{1}{2 \pi} \int_{\mathbb{R}} \mathrm{d} \omega \tilde{X}(\omega) \mathrm{e}^{-\mathrm{i} \omega t}
$$

with $\omega \in \mathbb{R}$.

\section{A.2. Parseval's theorem}

If $X(t)$ and $Y(t)$ are two time-dependent square-integrable functions, Parseval's theorem states

$$
\int_{\mathbb{R}} \mathrm{d} t X(t) \bar{Y}(t)=\frac{1}{2 \pi} \int_{\mathbb{R}} \mathrm{d} \omega \tilde{X}(\omega) \overline{\widetilde{Y}}(\omega),
$$

where the bar - denotes complex conjugation.

\section{Appendix B. Non-isothermal colloidal suspensions}

In this appendix we present explicit expressions for the Hamiltonian of a suspension of $N_{\mathrm{c}}$ rigid isotropic colloidal (c) particles that are suspended in a solvent (s) consisting of $N_{\mathrm{s}} \gg N_{\mathrm{c}}$ atomic or molecular isotropic particles as well as for the relevant variables of this system (see section 4.4.1), since these expressions are needed for the application of the MZFT. The phase-space variables of this system are the positions $\vec{r}_{i}^{\mathrm{c}}(t)$ and translational momenta $\vec{p}_{i}^{\mathrm{c}}(t)$ with $i=1, \ldots, N_{\mathrm{c}}$ of the colloidal particles as well as the positions $\vec{r}_{i}^{\mathrm{s}}(t)$ and momenta $\vec{p}_{i}^{\mathrm{s}}(t)$ with $i=1, \ldots, N_{\mathrm{s}}$ of the solvent particles at time $t$. In general, the colloidal and solvent particles are subjected to external potentials $U_{1}^{\mathrm{c}}(\vec{r})$ and $U_{1}^{\mathrm{s}}(\vec{r})$, respectively. Interactions between the particles are taken into account by the pair-interaction potentials $U_{2}^{\text {(cc) }}\left(\left\|\vec{r}-\vec{r}^{\prime}\right\|\right)$, $U_{2}^{(\mathrm{cs})}\left(\left\|\vec{r}-\vec{r}^{\prime}\right\|\right)$, and $U_{2}^{(\mathrm{ss})}\left(\left\|\vec{r}-\vec{r}^{\prime}\right\|\right)$ for colloid-colloid, colloid-solvent, and solvent-solvent particle interactions, respectively.

If $m_{\mathrm{c}}$ denotes the mass of a colloidal particle and $m_{\mathrm{s}}$ the mass of a solvent particle, the system is completely described by its Hamiltonian $[8,11]$

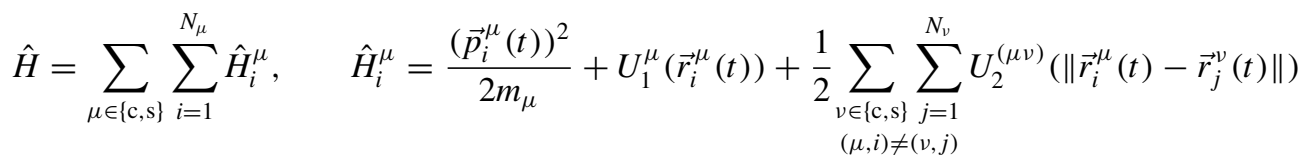


or equivalently by the Liouvillian

$$
\hat{\mathcal{L}}=\sum_{\mu \in\{\mathrm{c}, \mathrm{s}\}} \sum_{i=1}^{N_{\mu}}\left(\vec{\nabla}_{\vec{p}_{i}^{\mu}} \hat{H}\right) \cdot \vec{\nabla}_{\vec{r}_{i}^{\mu}}-\sum_{\mu \in\{\mathrm{c}, \mathrm{s}\}} \sum_{i=1}^{N_{\mu}}\left(\vec{\nabla}_{\vec{r}_{i}^{\mu}} \hat{H}\right) \cdot \vec{\nabla}_{\vec{p}_{i}^{\mu}}
$$

Conserved relevant variables of this system are the local concentration of colloidal particles $\hat{c}(\vec{r}, t)$ and the internal energy density $\hat{\varepsilon}(\vec{r}, t)$ with the microscopic expressions $[8,11]$

$$
\begin{aligned}
& \hat{c}(\vec{r}, t)=\sum_{i=1}^{N_{\mathrm{c}}} \delta\left(\vec{r}-\vec{r}_{i}^{\mathrm{c}}(t)\right), \\
& \hat{\varepsilon}(\vec{r}, t)=\sum_{\mu \in\{\mathrm{c}, \mathrm{s}\}} \sum_{i=1}^{N_{\mu}} \hat{H}_{i}^{\mu} \delta\left(\vec{r}-\vec{r}_{i}^{\mu}(t)\right) .
\end{aligned}
$$

The dynamics of these relevant variables is given by

$$
\dot{\hat{c}}(\vec{r}, t)+\vec{\nabla}_{\vec{r}} \cdot \hat{\vec{J}}^{c}(\vec{r}, t)=0, \quad \dot{\hat{\varepsilon}}(\vec{r}, t)+\vec{\nabla}_{\vec{r}} \cdot \hat{\vec{J} \varepsilon}(\vec{r}, t)=0
$$

with the local currents $\hat{\vec{J}}^{c}(\vec{r}, t)$ and $\hat{\overrightarrow{J^{\varepsilon}}}(\vec{r}, t)$. From the Hamiltonian (B.1) explicit expressions can be derived for these currents. They are $[8,11]$

$$
\begin{aligned}
\hat{\vec{J}^{c}}(\vec{r}, t)= & \sum_{i=1}^{N_{\mathrm{c}}} \frac{\vec{p}_{i}^{\mathrm{c}}(t)}{m_{\mathrm{c}}} \delta\left(\vec{r}-\vec{r}_{i}^{\mathrm{c}}(t)\right), \\
\hat{\overrightarrow{J^{\varepsilon}}}(\vec{r}, t)= & \sum_{\mu \in\{\mathrm{c}, \mathrm{s}\}} \sum_{i=1}^{N_{\mu}} \frac{\vec{p}_{i}^{\mu}(t)}{m_{\mu}} \hat{H}_{i}^{\mu}(t) \delta\left(\vec{r}-\vec{r}_{i}^{\mu}(t)\right)-\frac{1}{4} \sum_{\mu, v \in\{\mathrm{c}, \mathrm{s}\}} \sum_{\substack{i=1 \\
(\mu, i) \neq(\nu, j)}}^{N_{\mu}} \sum_{j=1}^{N_{v}} \frac{\vec{r}_{i j}^{(\mu \nu)}(t) \otimes \vec{r}_{i j}^{(\mu \nu)}(t)}{\left\|\vec{r}_{i j}^{(\mu \nu)}(t)\right\|} \\
& \times\left(\frac{\vec{p}_{i}^{\mu}(t)}{m_{\mu}}+\frac{\vec{p}_{j}^{v}(t)}{m_{\nu}}\right) \frac{\mathrm{d} U_{2}^{(\mu \nu)}(r)}{\mathrm{d} r} \int_{0}^{1} \mathrm{~d} \lambda \delta\left(\vec{r}-\vec{r}_{i}^{\mu}(t)+\lambda \vec{r}_{i j}^{(\mu \nu)}(t)\right)
\end{aligned}
$$

with the dyadic product $\otimes$ and the notation $\vec{r}_{i j}^{(\mu \nu)}(t)=\vec{r}_{i}^{\mu}(t)-\vec{r}_{j}^{\nu}(t)$.

\section{References}

[1] Reichl L E 1998 A Modern Course in Statistical Physics 2nd edn (New York: Wiley)

[2] Martin P C, Parodi O and Pershan P S 1972 Phys. Rev. A 62401

[3] Groot S R d and Mazur P 1984 Non-Equilibrium Thermodynamics 1st edn (Dover Books on Physics and Chemistry) (New York: Dover)

[4] Mori H 1965 Prog. Theor. Phys. 33423

[5] Zwanzig R and Mountain R D 1965 J. Chem. Phys. 434464

[6] Forster D 1974 Ann. Phys. 84505

[7] Grabert H 1982 Projection Operator Techniques in Nonequilibrium Statistical Mechanics 1st edn (Springer Tracts in Modern Physics vol 95) (Berlin: Springer)

[8] Forster D 1990 Hydrodynamic Fluctuations, Broken Symmetry, and Correlation Functions 1st edn (Advanced Book Classics vol 10) (New York: Perseus)

[9] Dhont J K G 1996 An Introduction to Dynamics of Colloids 1st edn (Studies in Interface Science vol 2) (Amsterdam: Elsevier)

[10] Zwanzig R 2001 Nonequilibrium Statistical Mechanics 3rd edn (New York: Oxford University Press)

[11] Wittkowski R, Löwen H and Brand H R 2012 J. Chem. Phys. 137224904

[12] Kadanoff L P and Martin P C 1963 Ann. Phys. 24419

[13] Marconi U M B and Tarazona P 1999 J. Chem. Phys. 1108032

[14] Marconi U M B and Tarazona P 2000 J. Phys.: Condens. Matter 12413

[15] Archer A J and Evans R 2004 J. Chem. Phys. 1214246

[16] Español P and Löwen H 2009 J. Chem. Phys. 131244101 
[17] Wittkowski R and Löwen H 2011 Mol. Phys. 1092935

[18] Wittkowski R, Löwen H and Brand H R 2010 Phys. Rev. E 82031708

[19] Wittkowski R, Löwen H and Brand H R 2011 Phys. Rev. E 83061706

[20] Wittkowski R, Löwen H and Brand H R 2011 Phys. Rev. E 84041708

[21] Emmerich H, Löwen H, Wittkowski R, Gruhn T, Tóth G I, Tegze G and Gránásy L 2012 Adv. Phys. 61665

[22] Grmela M and Öttinger H C 1997 Phys. Rev. E 566620

[23] Öttinger H C and Grmela M 1997 Phys. Rev. E 566633

[24] Jarzynski C 1997 Phys. Rev. Lett. 782690

[25] Assoud L, Ebert F, Keim P, Messina R, Maret G and Löwen H 2009 Phys. Rev. Lett. 102238301

[26] Löwen H 2010 J. Phys.: Condens. Matter 22364105

[27] Kahl G and Löwen H 2009 J. Phys.: Condens. Matter 21464101

[28] Löwen H, Bechhoefer J and Tuckerman L S 1992 Phys. Rev. A 452399

[29] Ivlev A V, Löwen H, Morfill G E and Royall C P 2012 Complex Plasmas and Colloidal Dispersions: ParticleResolved Studies of Classical Liquids and Solids 1st edn (Series in Soft Condensed Matter vol 5) (Singapore: World Scientific)

[30] Löwen H, Allahyarov E, Ivlev A and Morfill G E 2012 J. Phys.: Condens. Matter 24284125

[31] Pleiner H and Brand H R 1996 Pattern Formation in Liquid Crystals 1st edn (Partially Ordered Systems vol 9) ed A Buka and L Kramer (New York: Springer) chapter 2, pp 15-67

[32] Graham R and Haken H 1971 Z. Phys. 245141

[33] Graham R and Haken H 1971 Z. Phys. 243289

[34] Risken H 1972 Z. Phys. 251231

[35] Risken H 1996 The Fokker-Planck Equation: Methods of Solution and Applications 3rd edn (Springer Series in Synergetics vol 18) (Berlin: Springer)

[36] Hohenberg P C and Martin P C 1965 Ann. Phys. 34291

[37] Brand H, Dörfle M and Graham R 1979 Ann. Phys. 119434

[38] Callen H B and Welton T A 1951 Phys. Rev. 8334

[39] Chaikin P M and Lubensky T C 1995 Principles of Condensed Matter Physics 1st edn (Cambridge: Cambridge University Press)

[40] Elder K R, Provatas N, Berry J, Stefanovic P and Grant M 2007 Phys. Rev. B 75064107

[41] van Teeffelen S, Backofen R, Voigt A and Löwen H 2009 Phys. Rev. E 79051404

[42] Oxtoby D 1991 Liquids, Freezing and Glass Transition: Proc. Les Houches Summer School Course LI (USMG, NATO Advanced Study Institute, 3-28 July, 1989) vol 1, ed J-P Hansen, D Levesque and J Zinn-Justin (Amsterdam: North-Holland) pp 145-92

[43] Singh Y 1991 Phys. Rep. 207351

[44] Löwen H 1994 Phys. Rep. 237249

[45] Khalatnikov I M 1989 An Introduction to the Theory of Superfluidity 2nd edn (Frontiers in Physics vol 23) (Redwood City, CA: Addison-Wesley)

[46] Hohenberg P C and Halperin B I 1977 Rev. Mod. Phys. 49435

[47] Dohm V 1985 Z. Phys. B 6061

[48] Dohm V 1985 Z. Phys. B 61193

[49] Dohm V 1993 Phys. Scr. T49A 46

[50] Ahlers G 1980 Rev. Mod. Phys. 52489

[51] Tam W Y and Ahlers G 1985 Phys. Rev. B 325932

[52] de Gennes P-G 1971 Mol. Cryst. Liq. Cryst. 12193

[53] Liu M 1979 Phys. Rev. A 192090

[54] Brand H and Swift J 1983 J. Phys. Lett. 44333

[55] Cajas C A, Swift J B and Brand H R 1983 Phys. Rev. A 28505

[56] Cajas C A, Swift J B and Brand H R 1984 Phys. Rev. A 301579

[57] Brand H R 1986 Mol. Cryst. Liq. Cryst. Lett. 3147

[58] Liu M 1975 Phys. Rev. Lett. 351577

[59] Graham R and Pleiner H 1975 Phys. Rev. Lett. 34792

[60] Brand H R, Pleiner H and Renz W 1990 J. Phys. 511065

[61] Temmen H, Pleiner H, Liu M and Brand H R 2000 Phys. Rev. Lett. 843228

[62] Temmen H, Pleiner H, Liu M and Brand H R 2001 Phys. Rev. Lett. 86745

[63] Pleiner H, Liu M and Brand H R 2000 Rheol. Acta 39560

[64] Pleiner H, Liu M and Brand H R 2002 Rheol. Acta 41375

[65] Pleiner H, Liu M and Brand H R 2004 Rheol. Acta 43502

[66] Anero J G, Español P and Tarazona P 2013 J. Chem. Phys. 139034106

[67] Schmidt M 2011 Phys. Rev. E 84051203 